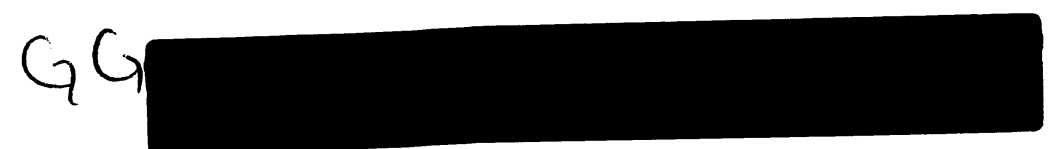

CERN LIBRARIES, GENEVA

CERN - CN 90-17

03 JUL. 1990

$c^{2}$

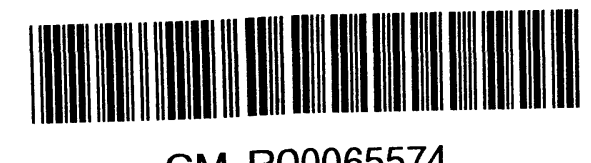

CERN-COMPUTING \& NETWORKS DIVISION

$\mathrm{CN} / 90 / 17$

CM-P00065574

June 1990

\title{
KUIP: the User Interface of PAW
}

Pietro Zanarini

Lecture given at the

Eighth Summer School on Computing Techniques in Physics, Skalsky Dvur, Czechoslovakia,

September 1989 


\section{Abstract}

KUIP is the User Interface system developed at CERN in the context of the PAW system. The basis of KUIP is the so-called Command Definition File (CDF), an application dependent text file which constitutes a very concise formal description of the application command structure. An on line help facility is integral part of the system (contained in the CDF but also obtainable dynamically from the application), as well as an automatic generation of written documentation in various text processing formats. Different dialogue modes are available, allowing commands to be entered by the user either in command mode (keyboard command lines) or in various menu modes (alphanumeric and graphics menus); switching from one dialogue style to another is possible at any moment during the interactive session. KUIP is implemented as a FORTRAN-77 library package (plus a utility program for the application developer, the so-called KUIP Compiler) on a wide range of host machines and operating systems, portability and generality being therefore its most valuable features. 


\section{Contents}

Chapter 1. Purpose of a User Interface Management System _ . . . . . . . . 1

Chapter 2. The KUIP system $\ldots \ldots \ldots \ldots \ldots \ldots \ldots$

Chapter 3. User interface $\ldots \ldots \ldots \ldots \ldots \ldots \ldots \ldots \ldots \ldots$

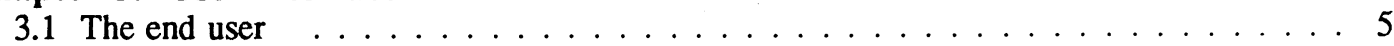

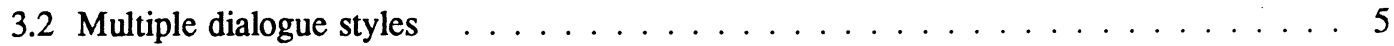

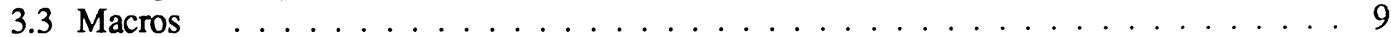

3.4 Vectors . . . . . . . . . . . . . . . . . . . . 9

3.5 Aliases and system functions $\ldots \ldots \ldots \ldots \ldots$

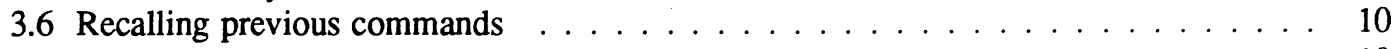

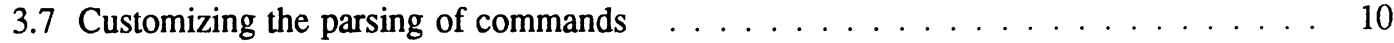

3.8 The KUIP command structure . . . . . . . . . . . . . . . . . . . . . . . . . . 10

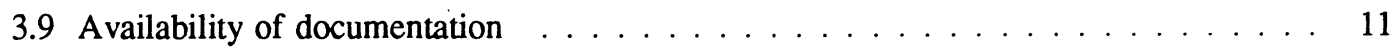

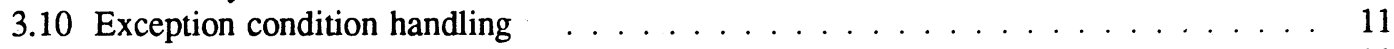

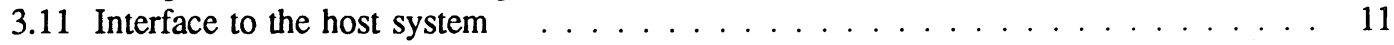

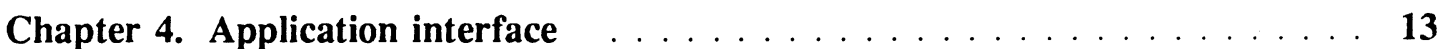

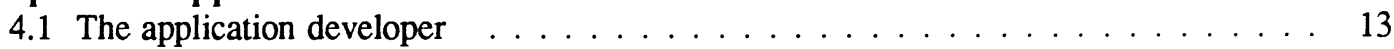

4.2 Command Definition File (CDF) . . . . . . . . . . . . . . . . . 13

4.3 Getting parameters in action routines $\ldots \ldots \ldots \ldots \ldots \ldots \ldots$

4.4 Implementation . . . . . . . . . . . . . . . . . 15

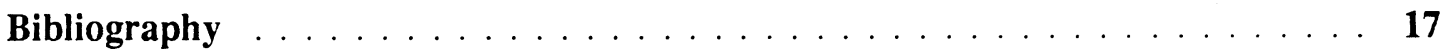

\section{Figures}

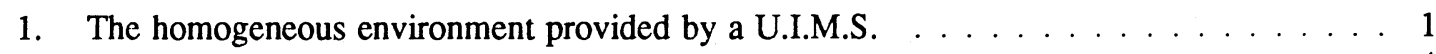

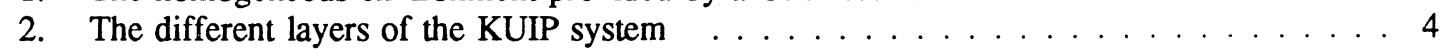

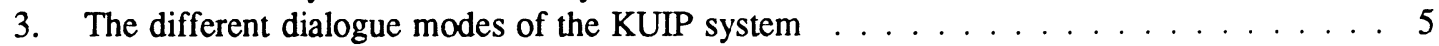

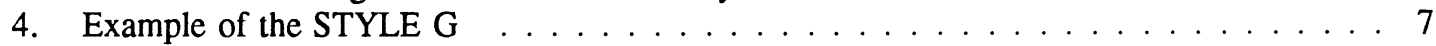

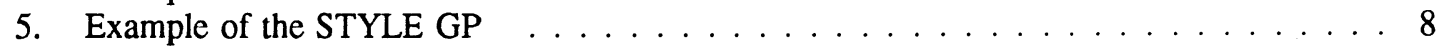

6. Building an application starting by its $\mathrm{CDF} \ldots \ldots \ldots \ldots \ldots \ldots$ 


\section{Chapter 1. Purpose of a User Interface Management System}

A User Interface Management System (UIMS) is a software toolkit intended to: provide a homogeneous environment in which different kinds of end users interact with different kinds of applications (see Figure 1)

provide some development tools to help application developers in making interactive an application program

A good UIMS design should achieve the best compromise between the ease of use (e.g. including maximum of online help and minimum of written documentation) and the avoidance of frustration of experienced users (e.g. not restricting only to graphics menus and not insisting on deep menu hierarchies).

Hetherogeneous users and applications require a multi-modal dialogue, i.e. different dialogue styles switchable interactively, like command line mode plus graphics and non-graphics menu modes.

Another important point is the mixed control facility, i.e. the possibility to have either the command processor (master) controlling the application (slave) or viceversa. The first condition can be encountered in the classical endless loop where the command processor ask the user to enter a new command line (or to click on menus). The second condition happens when the application program decides whether to give the control to the user (through the command processor) or ask directly the command processor to execute some given commands.

The coherence of the system is also another aspect that should not be neglected.
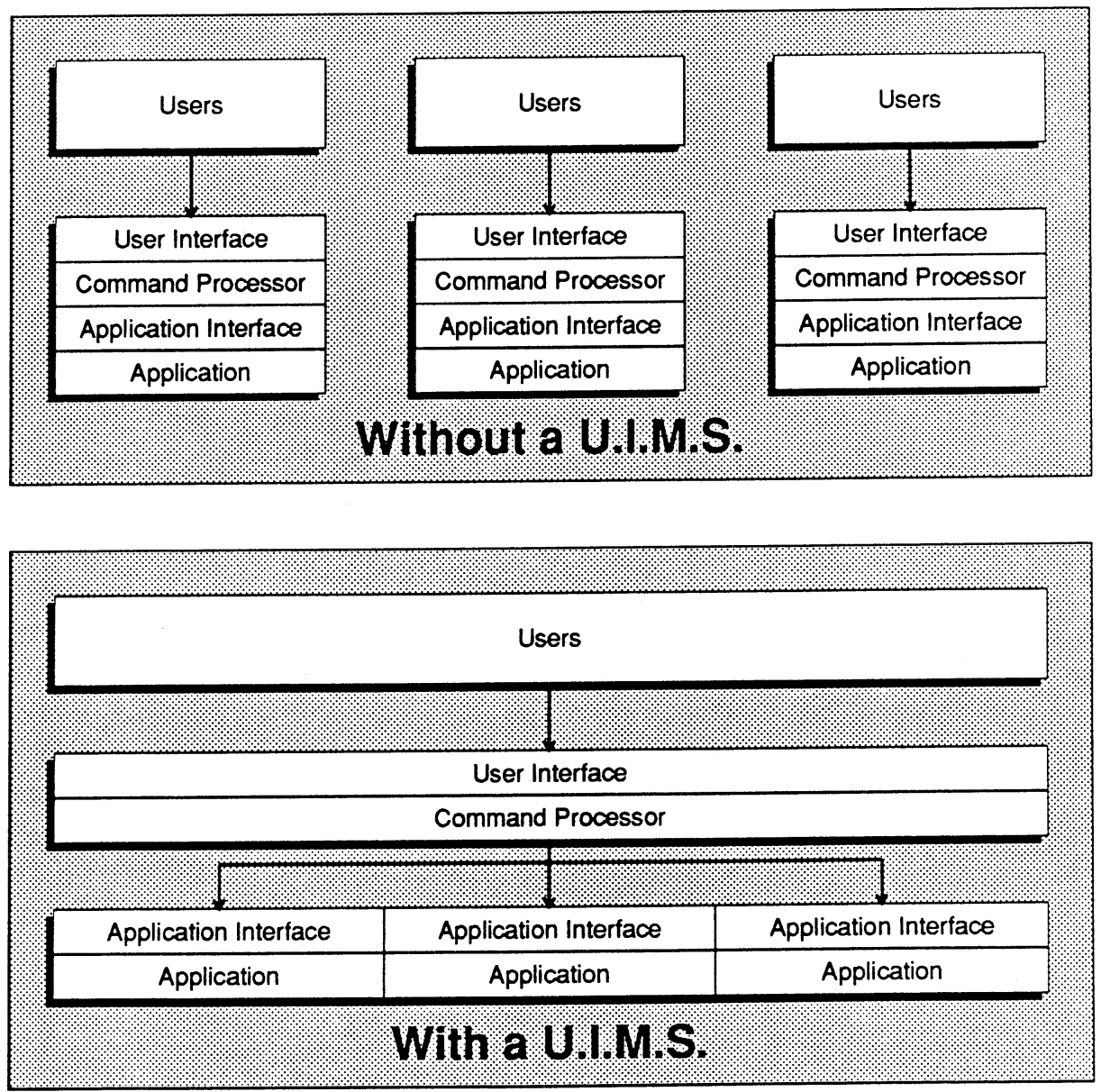

Figure 1. The homogeneous environment provided by a U.I.M.S. 


\section{Chapter 2. The KUIP system}

KUIP (Kit for a User Interface Package) [5] is the User Interface system developed at CERN in the context of PAW, the Physics Analysis Workstation system.[1][3][4]

Figure 2 shows the different layers present in the KUIP system.

KUIP represents a new approach to the relatively old problem of general-purpose User Interface systems. The basis of KUIP is the so-called Command Definition File (CDF), which constitutes a very concise formal description of the command structure. An appropriate module, the KUIP Compiler, generates from this file a set of FORTRAN subroutines, which are then compiled and linked with the interactive application.

The dialogue between the user and the system is handled either by typing command lines or by choosing from alphanumeric or graphical menus. In order to avoid verbosity in typing command lines, often useless and annoying for the experienced user, command abbreviations are possible as long as they do not produce ambiguities. Application commands are represented by an inverted tree, in analogy with file names of a Unix file system. The formal description of such a command structure is contained in the Command Definition File (CDF), a text file which can be considered as the basis of any KUIP-based application.

In command lines, path elements (branches and leafs) may be abbreviated, and even omitted (branches only), as long as the resulting command verb is not ambiguous. When using menu modes, the user has just to scan through the pull-down menus of the command tree.

The user is able to switch from one dialogue mode to another at any moment. Furthermore, menus do not need any special additional programming: they are automatically derived from the command structure as described in the CDF.

Needless to say, it is possible to group commands in 'macro' files and to invoke these sets of commands for repeated execution. Assignment and control statements may be used inside macros.

KUIP keeps track of all command lines entered (independently of the dialogue mode), which are automatically recorded in a macro file, and therefore may be edited and re-executed as with normal macros. A Unix-like history mechanism is also available.

An on line help facility is integral part of the system. This can be considered as a good step in the direction of the "system to be used without a manual". Also the importance of proper documentation should never be underestimated: it is vital to make available users' documentation since the release of the first prototype of the package (documentation which could be, if possible, produced automatically by the system itself). It is also very important to provide different levels of documentation: beginners' guide, users' guide, reference manual, each one having a different target and different users. One of the major problems normally encountered in this area is how to keep the documentation up-to-date. KUIP solved this problem by introducing a feature in the system which permits the automatic generation of documentation (already in text processing format). As an additional benefit, as the documentation is integral part of the language description system, the updating of the documentation is automatic.

The system has to be considered completely open-ended, in the sense that the user is able not only to personalize his own version, for instance using the mechanism of the KUIP macros, but he is also able to create his own interactive language adapted to his particular application, by modifying the Command Definition File. 


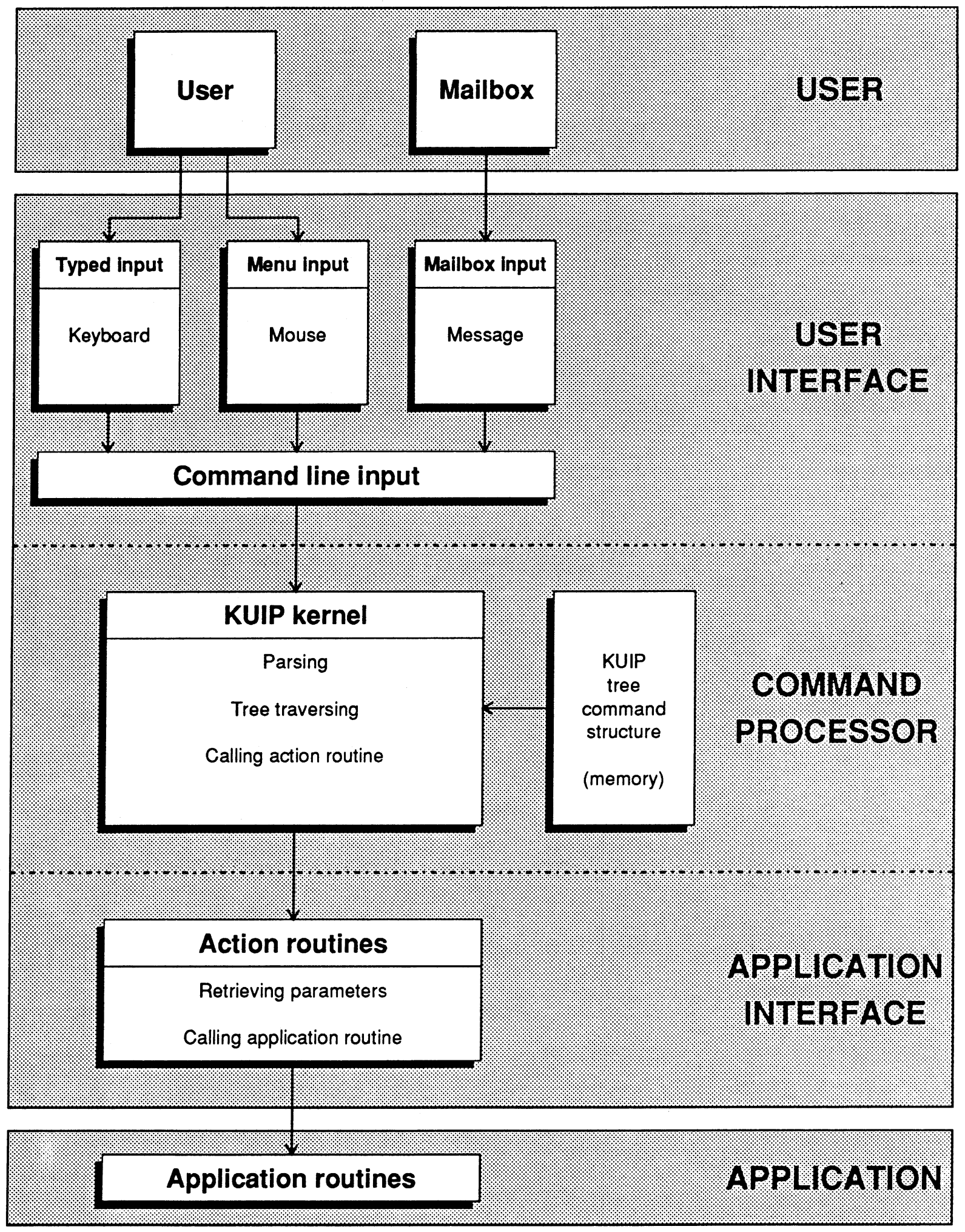

Figure 2. The different layers of the KUIP system 


\section{Chapter 3. User interface}

\subsection{The end user}

The end user is prompted by KUIP and asked for the next command, possibly with associated parameters. A check of the command syntax is performed and, if an existing command is referenced, the relevant application routine is called and parameters (if any) are fetched. Additional checks are performed on parameters, for type consistency (real, integer or character string), range correctness (with numeric parameters), etc.

Different dialogue modes (or styles) are available, by which commands can be entered by the user: from the default command mode (keyboard command lines) to various menu modes (alphanumeric and graphics pull-down menus). Switching from one dialogue style to another is possible at any moment during the interactive session: this is what makes a User Interface system suitable to heterogenous applications and to users with different tastes and knowledge of the application behind it.

\subsection{Multiple dialogue styles}

The dialogue style can be changed interactively (i.e. while executing the application program) with the command STYLE.

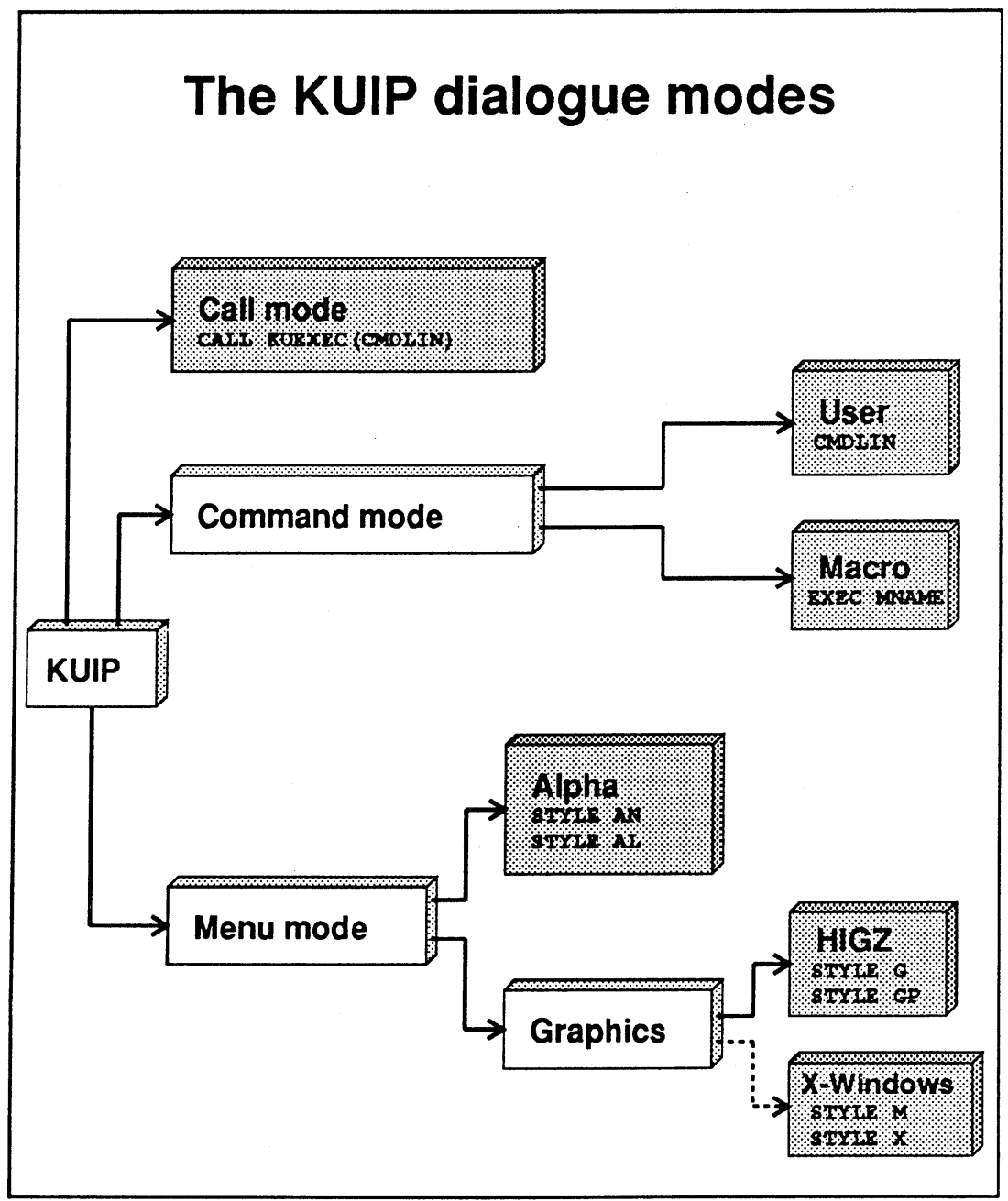

Figure 3. The different dialogue modes of the KUIP system 


\section{Command line mode}

In command mode the user enters a command line via the terminal keyboard.

The general syntax of an input command_line is a command_name optionally followed by a parameter_list. The command_name and parameter_list are separated by one or more blanks (therefore, no blanks should appear within the command_name). Using the regular expressions notation:

command_line $::=$ command_name (blank+ parameter_list)?

where the postfix unary operator ' + ' means 'one or more instances of the postfixed item' and '?' means 'zero or one instances of the postfixed item'. The parameters in the parameter_list are again separated by one or more blanks:

parameter_list $::=$ parameter (blank+ parameter)*

where '*' means 'zero or more instances of the postfixed item'. No blanks should then appear within a parameter, unless the whole parameter is enclosed in single quotes, like for example 'This parameter has blanks' or the blank filled parameter ' '.

The command name is a structured name representing the path along the inverted tree structure handled by KUIP. Each element of the path, called command_element, is separated from the others by one slash:

command_name $::=$ command_element ( / command_element )*

The rightmost command_element of a command_name must be a leaf of the tree, i.e. a terminal command_element, while the others are considered menus. The command_name can have up to 10 levels of command elements (i.e. 9 levels of menus).

A command can always be abbreviated, as long as it does not become ambiguous with other commands, by:

Omitting leftmost command elements

Omitting rightmost characters of a command element

The shortest unambiguous abbreviation for any command is not fixed, but depending on the whole command tree structure: KUIP takes care to list all possible ambiguities should the user have entered an ambiguous command.

The list of all executable commands can be obtained just by typing one slash. This is a command line having a null command element both to the left and to the right of the separator slash; by definition a null command element is ambiguous with every non-null command element, therefore all the available commands will be listed as possible ambiguities.

As explained above, a command line consists of a command part optionally followed by a parameter part.

Parameters can be mandatory or optional. An optional parameters always has a default value, which is used when the user does not specify the parameter.

The order of parameters in the command line is important and must match the semantic definition of the command. Mandatory parameters are always specified before any optional parameters. 


\section{Menu modes}

In addition to the command line mode, KUIP also allows various menu modes:

Alphanumeric, entered by STYLE AN or STYLE AL.

Selection is from a list by Number or by Letter. This mode still uses the terminal keyboard as input, but the user, instead of typing directly the full command, is guided into the tree of the command structure which is scanned through its branches until a leaf is found. The resulting path name corresponds then to the command which may be invoked.

Graphics: This mode is particularly interesting for workstations. It should not be used with dumb terminals.

- Pull-down menus, entered by STYLE G; fixed layout, reflecting the command structure

- Panels of function keys, entered by STYLE GP; interactive user definable multiple layouts.

\section{Style G(raphics)}

Under STYLE G the user is still guided through the command tree as for the alphanumeric styles, but the mouse is used to navigate on pull down menus in the graphics window. The shadowed part is available for the graphics application.

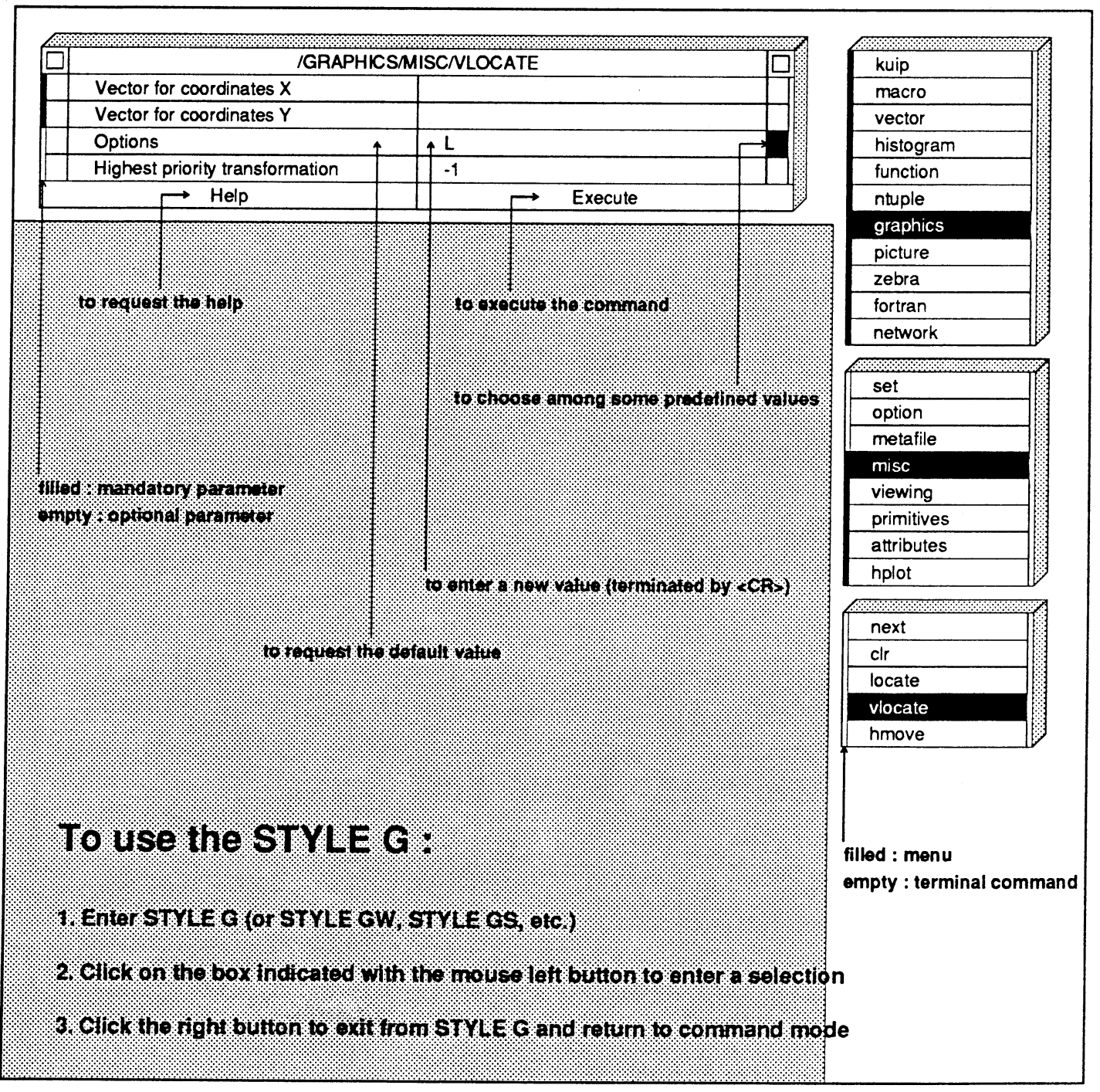

Figure 4. Example of the STYLE G 


\section{Style G(raphics) P(anels)}

Panels of graphics function keys may be defined with the command PANEL:

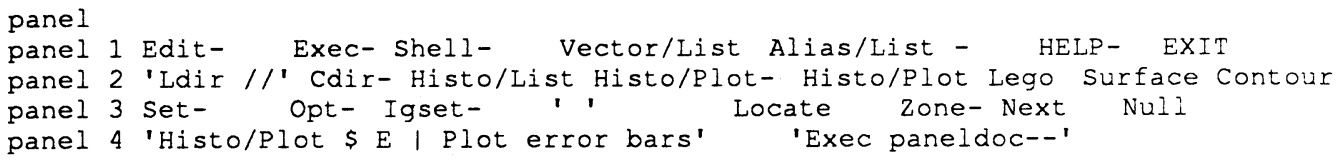

Then enter STYLE GP, move the mouse to a graphics function key and click the left mouse button; the text written on the key will be sent for execution as if it were typed in via the keyboard. Parameters can also be added interactively by the user.

A panel can be redefined from within itself. For example by selecting the key Exec paneldoc-and entering the number 1 , the macro file paneldoc1.kumac will be used as input and executed. If it contains the following lines:

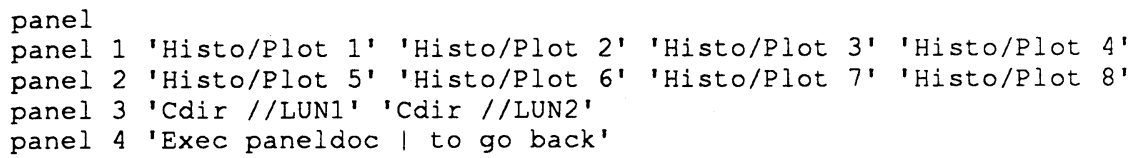

then the panel will be modified accordingly.

Both STYLE G and STYLE GP are based on the IGMENU routine of the HIGZ [2] package.

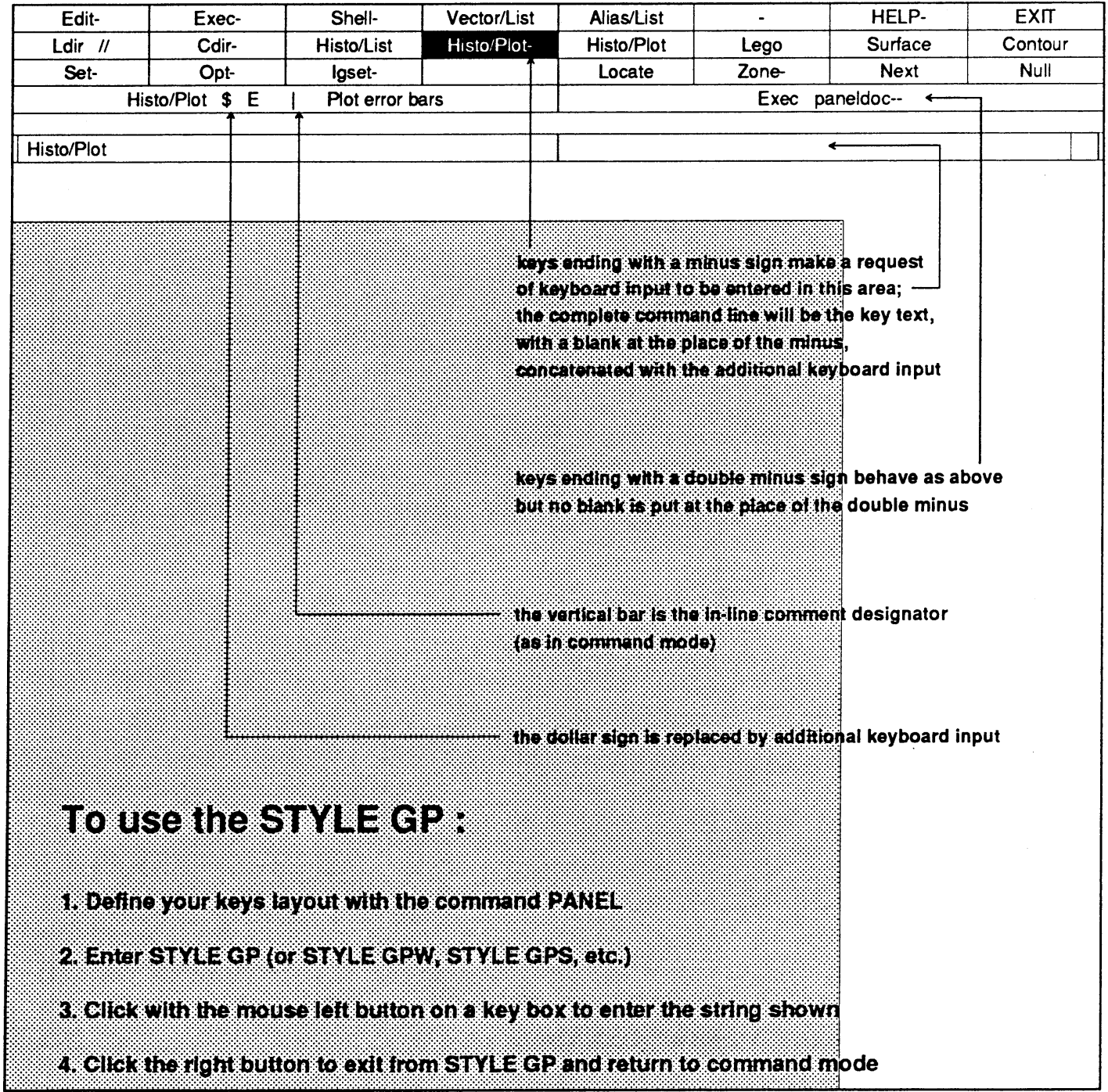

Figure 5. Example of the STYLE GP 


\subsection{Macros}

A macro is a set of command lines stored in a file, which can be created/edited with the command EDIT and executed with EXEC: EXEC FILE_NAME executes the command lines contained in the file FILE_NAME. A macro file can contain several macros, which can be identified by following the file name with a dash sign and the name of the macro, like for example: EXEC FILE_NAME\#MACRO_NAME

In addition to all available commands, some special 'macro statements' are available inside macros, like for instance IF, GOTO, ON ERROR GOTO, READ, variable assignment, etc.

Positional and non-positional (i.e. named) parameters can be passed to the invoked macros.

\subsection{Vectors}

Vectors are named arrays of numerical data, memory resident, whose content can be by entered directly by the user, or read from disk files. At the end of an interactive session, they are lost, unless previously saved onto disk files.

Vectors can have up to 3 dimensions (in fact they are 'arrays', called 'vectors' for historical reasons).

One interesting characteristic of vectors is the possibility to handle them either interactively, or in the application program, by means of KUIP routines which return the addresses of a given vector, thus making its content available to the application.

Simple arithmetic operations can be applied to vectors. In addition, if KUIP is linked with the SIGMA[8] array manipulation package, all the power of SIGMA is automatically available (through the system function \$SIGMA, described later).

\subsection{Aliases and system functions}

Aliases are defined to provide shortcut abbreviations for the input line or some part of it. An alias name can be any string of characters (excepted the single quote and the blank) and whenever encountered in an input line it will be literally replaced by its value (another string of characters).

While aliases have a fixed value, system functions can be seen as aliases whose value depends on the function name and on its arguments (if any). Therefore, also system functions are literally replaced by their current value whenever encountered in a KUIP command line. Names of system functions always start with a dollar sign, and some of them are followed by a parameter list enclosed in parentheses. System functions are mainly used inside macros.

Examples of system functions are:

\$DATE, giving current date

\$CPTIME, giving CP time elapsed since last call

\$VEXIST(VNAME), telling if a vector exists or no

\$VDIM(VNAME,IDIM), giving dimensions of a vector

\$SUBSTRING(STRING,IX,NCH), \$UPPER(STRING), etc., performing string handling

\$SIGMA(SIGMA_Expression), invoking SIGMA to evaluate an expression

\$ARGS, giving the arguments of the command line entered to run the program

etc. 


\subsection{Recalling previous commands}

In addition to the host machine local facilities in recalling previous commands, KUIP allows to:

Enter the command LAST which stores all (or some) commands typed in the session on a disk file (by default LAST.KUMAC) and invokes the local editor on the file.

The history file is updated automatically every 25 commands (but the rate can be changed with the command RECORDING) and at the end of a session. At the beginning of another session the old history file LAST.KUMAC is renamed into LAST.KUMACOLD and a new LAST.KUMAC is opened. In this way the user keeps always track of all the commands entered in the previous and in the current sessions. The history files contain also heading and trailing comment lines, showing the date and time at which the session was started/stopped.

The command LAST 0 MYFILE may be put in the user's logon macro file to define the name of the history files as MYFILE.KUMAC and MYFILE.KUMACOLD. This is useful to avoid sharing the same LAST.KUMAC file by several KUIP-based applications running on the same disk directory (e.g. PAW, GEANT[6], CMZ[7] , etc.)

Use a Unix C-shell-like history mechanism, starting a command with an exclamation mark followed by:

- An absolute number $\mathrm{n}$, to re-execute the $\mathrm{n}$-th command entered since the beginning of the session (e.g. !3)

- A minus sign and a relative number, to re-execute the command identified by the current command number minus $\mathrm{n}$ (e.g. !-2)

- Another exclamation mark, to re-execute the last command entered (e.g. !!)

- Anything else (i.e. a non-numeric string), to re-execute the latest command entered which starts with the specified string (e.g. !EXE)

- Nothing else, to show the list of recallable commands (e.g. just !)

To have the command numbering the prompt string should be defined as containing an open and closed brackets (e.g. by the command SET/PROMPT 'MYPROG []'), inside which KUIP will put the command number.

\subsection{Customizing the parsing of commands}

The command lines may be easily filtered by the user by COMMAND CHPATH, allowing a shorthand invokation of one frequently used command.

CHPATH is normally composed by a command and one or more 'token place-holders', indicated by $\$ n(n=1,2, \ldots, 9)$ for the $n$-th token, or $\$ *$, for the entire command line. The interpretation of a command line, once the user has entered a COMMAND CHPATH line is as follows:

if the command line contains a valid (not just ambiguous) command, then the command line is left unchanged, and processed as such.

if the command line does not contain a valid command, then it is interpreted as a sequence of one or more tokens (tokens are separated by blanks). The resulting command line will be the string defined by CHPATH, where the 'token place-holders' are replaced by the actual tokens given in the command line.

\subsection{The KUIP command structure}

As already mentioned, the command structure is represented by an inverted tree, like a Unix file system. This feature, compared to a flat command structure, presents the advantage of a cleaner representation through menus, especially when many commands are present in the structure.

For example, PAW (the first application that has used KUIP) has currently 212 different commands: it is hard to see how could one visualize those commands via graphics menus if all the commands were at the same level and not tree structured ! 
There is another advantage when groups of commands have to be merged. KUIP has, for instance, three menus of so-called system commands: KUIP, MACRO and VECTOR. The commands defined by the application can then be linked to those menus, if desired, or, as it is more frequent, can be grouped into one or more menus, in addition to the system menus.

The possibility of redefining the root of the tree for command scanning is also available. If, for instance, we have an application command called MYMENU/DELETE and we want be able to enter just DELETE to recall it, i.e. without getting the ambiguities from the other KUIP commands KUIP/ALIAS/DELETE and VECTOR/DELETE, we can specify a new root with the command ROOT MYMENU. From then on, the commands are first searched starting from the new root: if a command is found it is executed. Only if a command is not found a second pass of search is done, starting now from the top root of the tree.

\subsection{Availability of documentation}

There are two ways within KUIP to get help on the command structure, coming directly from the CDF. One can ask for either on-line help (with the command HELP), or on-line production of a text file containing the manual (with the command MANUAL), in plain format or in a text-formatted format using the most popular text formatting systems (SGML, TEX, LATEX, etc.).

\subsection{Exception condition handling}

User breaks (e.g. CTRL/C) are handled within KUIP (IBM excluded): when a break is issued the execution of the current command is aborted and KUIP is waiting again for the next command. Nevertheless, one has to be careful in using this facility, because it requires that the code underneath must be reentrant.

Program exception conditions (for example, floating-point overflow, negative square root, etc.) are also handled (IBM included): on the occurrence of the exception a warning is issued and the program flow is restarted again to wait for the next command, as for user breaks.

\subsection{Interface to the host system}

When running a KUIP-based application program, there are facilities to interact with the host system, namely:

Issue commands directed to the host

Create sub-processes

Invoke local editors

Handle user break and system fault interception. 


\section{Chapter 4. Application interface}

\subsection{The application developer}

The most tedious part of the application developer's job, i.e. the specification of all the commands with their associated parameters and action routines, as well as their relationships, becomes quite simple within KUIP because a non-procedural approach is used. Instead of calling many KUIP routines to define the full command structure, the application developer has only to provide a text file called Command Definition File (CDF), containing a formal description of the desired command structure, which has the form of an inverted tree. A special program, the so-called KUIP Compiler (KUIPC), has then to be invoked: it reads the CDF and generates a new file containing the necessary FORTRAN code (i.e. KUIP calls) to define the command structure as described into the CDF.

Therefore only very few KUIP routines have still to be called by the application developer: one in particular to transfer the control to the KUIP main input loop, plus a few more (in action routines) to fetch the parameters, if any, associated to the commands entered interactively by the end user.

This formal approach compared to a traditional procedural approach offers many advantages:

reduced probability of making mistakes in defining commands

increased readibility of the command structure

easy editing of the command structure:

- cutting and pasting parts

- merging with different CDF's

\subsection{Command Definition File (CDF)}

The command tree structure is fully described through a Command Definition File (CDF), a text file which is read by KUIPC, the KUIP Compiler, and converted into a new text file containing the FORTRAN code to be compiled and linked with the application to obtain the command structure as defined in the CDF. See Figure 6.

The CDF contains a full description of commands and parameters, namely:

Item name

Help text

Help routine

Action routine

Parameters

- name

- prompt string

- type (mandatory/optional + Real/Integer/Character-string)

- default value (optional)

- numerical range or set of suggested values (optional)

The lines starting with a '>' are control lines. In the following, we list an example of CDF (in fact it is the beginning of the PAW CDF) and the corresponding FORTRAN code generated by the KUIP Compiler. 


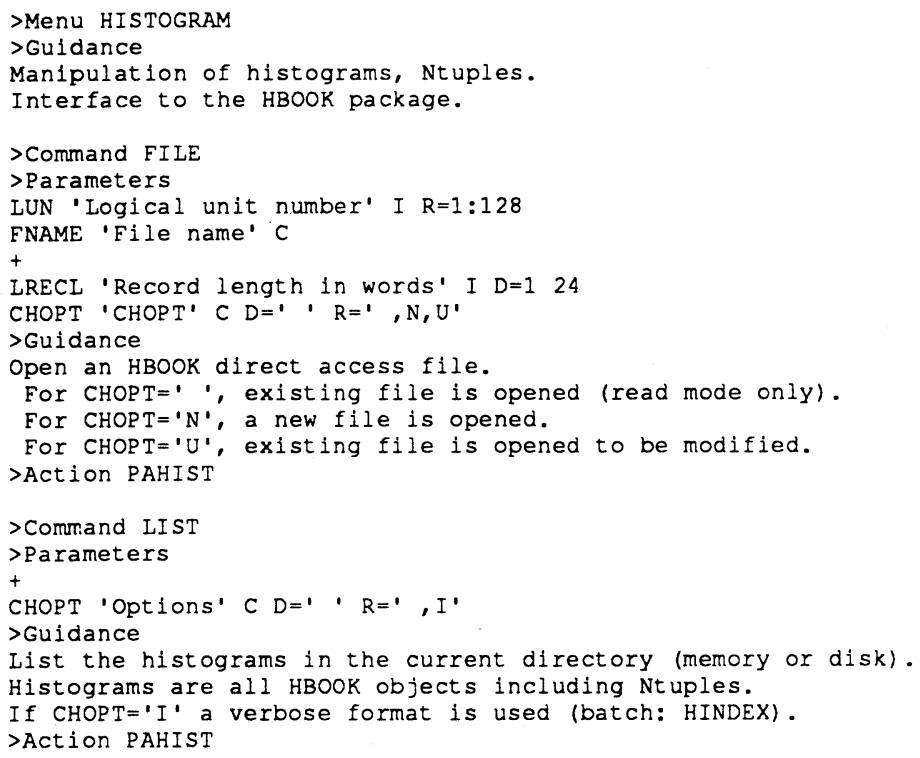

CALL KUNWG ( 52)

CALL KUCMD(' ', 'FILE', 'C'

CALL KUNDPV ( $1,1,1,1,1)$

CALL KUPAR('FILE', 'LUN','Logical unit number','I','S')

CALL KUPVAL ('FILE', 'LUN', $1, \ldots$, ' ', 'L')

CALI KUPVAL ('FILE', 'LUN', 128, .,' ' ', 'H')

CALL KUNDPV ( $1,1,1,1,1)$

CALI KUPAR('FILE', 'FNAME', 'File name', 'C', ' $S$ '

CALL KUNDPV ( $1,1,1,1,1)$

CALL KUPAR ('FILE', 'LRECL', 'Record length in words', 'IO', 'S')

CALL KUPVAL ('FILE', 'LRECL', $124, \ldots$ ' ', 'D')

CALL KUNDPV ( 1, 1, 1, 1, 2)

CALL KUPAR ('FILE', 'CHOPT', 'CHOPT', 'CO', 'S'

CALL KUPVAL ('FILE', 'CHOPT', , .,' ', 'D'

CALL KUPVAL ('FILE', 'CHOPT', , . ' ' N, U', 'V')

GUID ( 1)='Open an HBOOK direct access file."

GUID $(2)=1$ For CHOPT $=1, "$, existing file is opene"//

+'d (read mode only).'

$\operatorname{GUID}(3)=1$ For CHOPT$={ }^{\prime} \mathrm{N}$ '', a new file is opened.'

GUID ( 4)=' For CHOPT="U'", existing file is opene'//

+'d to be modified.'

CALL KUGUID ('FILE', GUID, 4, 'S')

CALI KUACT ('FILE', PAHIST)

CALL KUNWG ( 46)

CALL KUCMD(' ', 'LIST', 'C')

CALI KUNDPV ( 1, 1, 1, 1, 1)

CALL KUPAR/'LIST', 'CHOPT', 'Options', 'CO', 's'

CALL KUPVAL ('LIST', 'CHOPT', .,' ', D')

CALL KUPVAL ('LIST', 'CHOPT', , .,', I', 'V')

GUID( 1)='List the histograms in the current direc'//

+'tory (memory or disk).'

GUID( 2)='Histograms are all HBOOK objects includi"//

+ 'ng Ntuples.'

GUID ( 3)='If CHOPT="'I'" a verbose format is used $/ /$

+' (bat ch: HINDEX).

CALI KUGUID ('LIST', GUID, 3, 'S')

CALL KUACT ('LIST', PAHIST) 


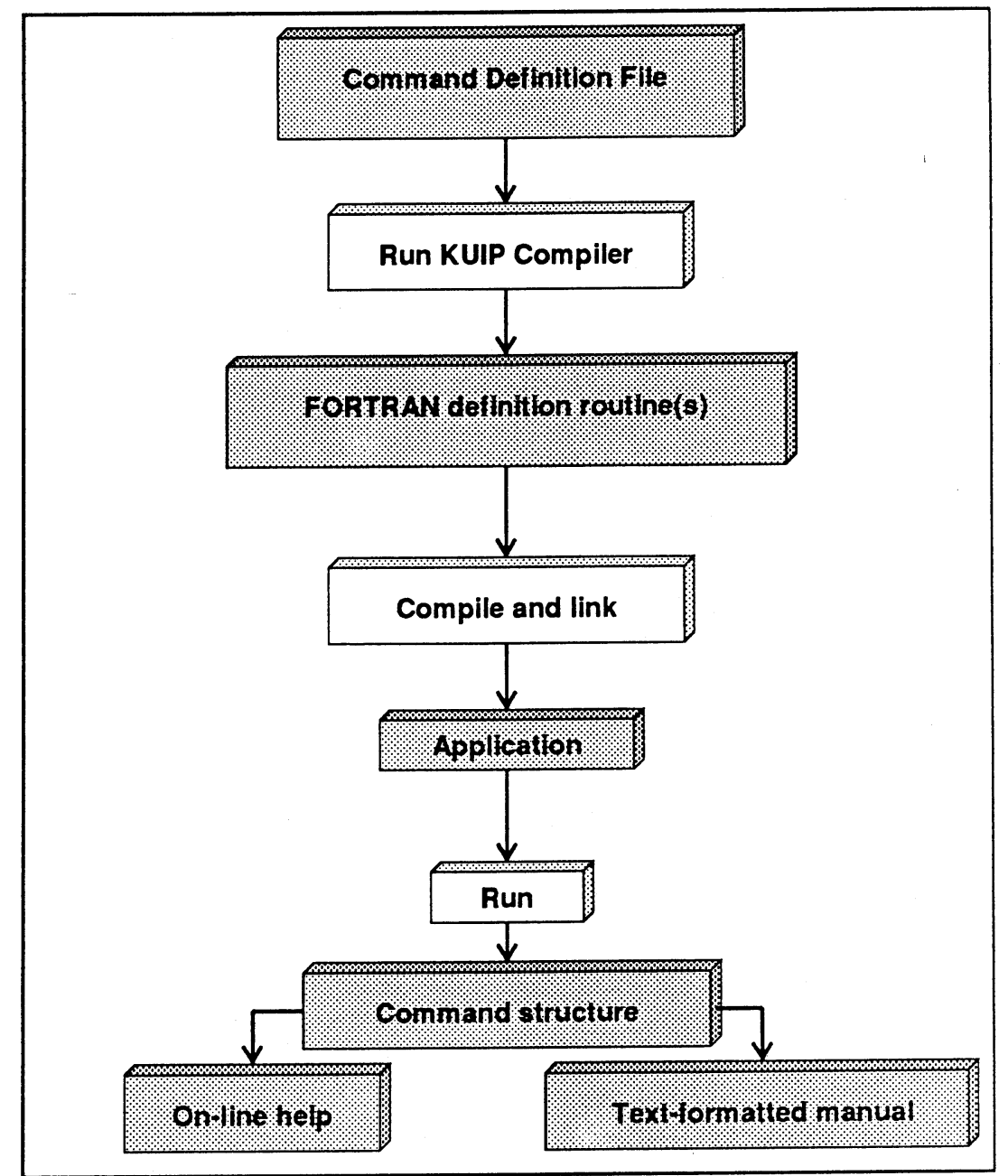

Figure 6. Building an application starting by its CDF

\subsection{Getting parameters in action routines}

Action routines are called automatically by KUIP when the corresponding command is entered. The application developer, in addition to defining their names with the >Action keyword in the $\mathrm{CDF}$, has to write these action routines and link them together with the rest of its application.

Inside action routines, parameters, as defined with >Parameters in the CDF, have to be retrieved by calling the KUGETx KUIP routines, e.g. KUGETI(IPAR), KUGETR(RPAR),

KUGETC(CHPAR,NCH), etc. for Integer, Real and Character type parameters respectively.

\subsection{Implementation}

KUIP has been written at CERN in FORTRAN-77, the standard language in High Energy Physics, and requires the standard CERN libraries.

It is implemented on a wide range of host machines and operating systems: IBM (VM/CMS and MVS/TSO), Cray (UNICOS), Vax and VaxStation (VMS and Ultrix), DEC Station (Ultrix), Apollo (Aegis and Unix), Sun (Unix), Silicon Graphics (Unix), IBM RS-6000 (AIX), etc.

Large CERN application programs are using KUIP: PAW, GEANT3, CMZ, just to mention some of them. 


\section{Bibliography}

[1] Bock R., Brun R., Couet O., Cremel-Somon N., Marin J.C., Nierhaus R., Pape L., Vandoni C., and Zanarini $P$.

PAW - Towards a Physics Analysis Workstation.

Computer Physics Communications 45, North-Holland, Amsterdam, 1987.

[2] Bock R., Brun R., Couet O., Cremel-Somon N., Nierhaus R., Vandoni C., and Zanarini P.. CERN Program Library Q120.

HIGZ - High level Interface to Graphics and ZEBRA.

1988.

[3] Brun R., Couet O., Vandoni C., and Zanarini P.

PAW, a general-purpose portable software tool for data analysis and presentation. Computer Physics Communications 57, North-Holland, Amsterdam, 1989.

[4] Brun R., Couet O., Vandoni C., and Zanarini P.. CERN Program Library Q121.

PAW - Physics Analysis Workstation. 1990.
[5] Brun R. and Zanarini P.. CERN Program Library I102. KUIP - Kit for a User Interface Package. 1988.

[6] Brun R., Bruyant F., Maire M., McPherson A.C., and Zanarini P..

CERN Data Handling Division, DD/EE/84-1. GEANT3.

1987.

[7] Brun M., Brun R., and Rademakers A.A. CMZ - A source code management system. Computer Physics Communications 57, North-Holland, Amsterdam, 1989.

[8] Hagedorn R., Reinfelds J., Vandoni C.E., and Van Hove L..

CERN Report 78-12.

SIGMA, A New Language for Interactive Array-oriented Computing. 1978. 